\title{
Preparation and Characterization of Silver Doped ZnO Nanostructures
}

\author{
Nguyen Van Nghia*, Tran Nam Trung, Nguyen Ngoc Khoa Truong, Doan Minh Thuy \\ Department of Physics, Quy Nhon University, Quy Nhon, Vietnam \\ Email: *nguyenvannghia@qnu.edu.vn
}

Received April 25, 2012; revised June 7, 2012; accepted July 3, 2012

\begin{abstract}
$\mathrm{ZnO}$ was prepared by hydrothermal method. The result of scanning electron microscopy showed that the materials had nano rod structures. Ag-doped $\mathrm{ZnO}$ was prepared by UV-photoreduction. Crystalline phases and optical absorption of the prepared Ag-doped $\mathrm{ZnO}$ samples were determined by X-ray diffraction, Raman spectrum, UV-visible, and UVphotoreduction spectrophotometer. X-ray analyses revealed that $\mathrm{Ag}$ was doped $\mathrm{ZnO}$ crystallizes in hexagonal wurtzite structure. The incorporation of $\mathrm{Ag}^{+}$in the site of $\mathrm{Zn}^{2+}$ provoked an increase in the size of nanocrystals as compared to pure $\mathrm{ZnO}$. The photocatalytic and photoluminescence properties of materials were considered.
\end{abstract}

Keywords: Nanostructures; Photocatalysis; Hydrothermal; ZnO; Silver Doping

\section{Introduction}

Nanostructured $\mathrm{ZnO}$ materials have received considerable interest from scientists due to their remarkable performance in electronics, optics and photonics. $\mathrm{ZnO}$ is a wide band gap ( $3.37 \mathrm{eV}$ at room temperature) compound semiconductor that is appropriate for short wavelength optoelectronics applications. The large exciton binding energy $(60 \mathrm{meV})$ in $\mathrm{ZnO}$ crystal allows efficient excitonic emission at room temperature. Therefore, $\mathrm{ZnO}$ nanostructures have had a wide range of high technology applications like surface acoustic wave filters, photonic crystals, gas sensors, photocatalysis [1-3]. Because of having a wide bandgap, $\mathrm{ZnO}$ can only be activated by ultraviolet light of wavelength below $385 \mathrm{~nm}$. The ultraviolet light reaching the earth's surface is less than $5 \%$ of the solar energy, which is too low to attain significant photodegradation in commercial application. Some interesting approaches have been adopted to extend the photoresponse of $\mathrm{ZnO}$ toward the visible spectral region, such as implanting transitional metal ions [4,5].

Metal silver is also a significant visible light photosensitizer, which is stable and nontoxic. Ag is also relatively cheap; thus Ag modification is of great significance for industrial practice. The improvement in efficiency of photocatalytic reactions under visible light is explained as the result of a vectorial transfer of photogenerated electrons and holes from metal to semiconductor. Moreover, $\mathrm{ZnO}$ modified by $\mathrm{Ag}$ can improve the distribution of surface charges, accept a conduction band generated by

*Corresponding author. solar light irradiation during photoreaction, prevent the recombination of the photogenerated electron-hole. Many researchers reported that $\mathrm{ZnO}$ thin film with $\mathrm{Ag}$ doping, which enhances ultraviolet emission and improves electrical and optical properties, was prepared by wet chemical [6], DC magnetron sputtering [7] and pulsed laser deposition [8].

In this work, silver nanoparticles were deposited on the surface of $\mathrm{ZnO}$ nanorods (prepared by hydrothermal method) by using a photochemical reduction under UV irradiation. We also have compared the photocatalytic properties of $\mathrm{Ag}$-doped $\mathrm{ZnO}$ and $\mathrm{ZnO}$ nanorods $(\mathrm{ZnO}$ NRs).

\section{Experimental}

\subsection{ZnO-NRs Preparation}

$4,6 \mathrm{~g}$ of zinc acetate $\left(\mathrm{Zn}(\mathrm{OAc})_{2}\right.$ powders were dissolved in $40 \mathrm{ml}$ a solvent etanol under stirring for $1 \mathrm{~h}$ (called M1 solution). The M2 solution was prepared by adding 2,5 $\mathrm{g}$ oxalic acid $\left(\mathrm{H}_{2} \mathrm{C}_{2} \mathrm{O}_{4}\right)$ into $40 \mathrm{ml}$ etanol under stirring. $\mathrm{M} 2$ was slowly poured in the M1 solution under ultrasonic wave $(35 \mathrm{kHz}, 100 \mathrm{~W})$ for 30 minutes. The sonicated solution was then moved to a teflon vessel and put in a stainless steel autoclave for carrying out the hydrothermal treatment at $140^{\circ} \mathrm{C}$ for 20 hours. The stainless steel was then opened at room temperature and the precipitates were separated and washed repeatedly by deionized water until the $\mathrm{pH}$ value of the washing solution became lower than 7. The final as-prepared product was dried under vacuum at temperature of $80^{\circ} \mathrm{C}$ for 12 hours. Then 
the product was calcined at temperature of $450^{\circ} \mathrm{C}$ for 1 hour in air.

\subsection{Ag-Doped ZnO Preparation}

0,5 g of $\mathrm{ZnO}-\mathrm{NRs}$ were dispersed into deionized water under stirring. The suspension was sonicated for $30 \mathrm{~min}$ by ultrasonic wave. After ultrasonically treated the suspension was further magnetically stirred for $30 \mathrm{~min}$ under UV irradiation. Then $0.007 \mathrm{~g} \mathrm{AgNO}_{3}$ were added into the suspension $\left(\mathrm{m}_{\mathrm{Ag}}: \mathrm{m}_{\mathrm{Zn}}=1 \%\right)$ and followed by UV illumination for $4 \mathrm{~h}$ under stirring. The black powder was centrifuged, rinsed with deionized water repeatedly to purify the product, and finally dried at $70^{\circ} \mathrm{C}$ under air for $5 \mathrm{~h}$. The product was denoted $\mathrm{Zn}-\mathrm{Ag}_{1}$.

Using the same method, we prepared products $\mathrm{Zn}-\mathrm{Ag}_{2}$, $\mathrm{Zn}-\mathrm{Ag}_{3}, \mathrm{Zn}-\mathrm{Ag}_{4}, \mathrm{Zn}-\mathrm{Ag}_{5}$. The more silver ion was doped, the darker in colour of the product was.

\subsection{Characterization and Measurement}

The morphology dimensions and microstructure measurements of the samples were performed using a Hitachi S4800 scanning electron microscope (SEM). The crystalline phases of the obtained samples were characterized by using a Siemens D-5005 X-ray powder diffractometer (XRD) with a monochromatized $\mathrm{Cu}-\mathrm{K} \alpha$ irradiation $(\lambda=$ $1.54056 \AA)$. The Raman spectra were recorded on a Nicolet spectrometer equipped with an optical microscope at room temperature. UV-Vis spectra were measured by a Scan UV-Vis spectrophotometer (Varian, Cary 500).

The photocatalytic activity was evaluated by measuring the decomposition of the aqueous solution of methylene blue (with a concentration of $10 \mathrm{mg} / \mathrm{L}$ ) under sunlight irradiation for $30 \mathrm{~min}$ of pure $\mathrm{ZnO}-\mathrm{NRs}$ and Ag-doped $\mathrm{ZnO}$. The reactor was equipped with water circulation in the outer jacket in order to maintain a constant temperature. Prior to irradiation, the suspensions were magnetically stirred in dark for $1 \mathrm{~h}$ to ensure an establishment of adsorption/desorption equilibrium. Then the solution was filtered to remove "particles". The resulted solution was analyzed by recording variations in the absorption in UV-visible spectra of methylene orange using a Shimadzu 1601-PC Ultraviolet-visible spectrometer.

\section{Results and Discussion}

The XRD patterns of $\mathrm{ZnO}-\mathrm{NRs}$ and $\mathrm{Zn}-\mathrm{Ag}_{5}$ are exhibited in Figure 1.

As can be seen from Figure 1, all the samples were well crystalline, and of hexagonal wurtzite phase (JCPDS file No. 36-1451). However, the Ag-doped samples revealed some additional diffraction peaks marked with "*" associated with the face-centered-cubic phase of me- tallic Ag (JCPDS file No. 04-0783). The appearance of Ag peaks in the diffraction patterns indicates clearly the formation of crystalline silver clusters in the nanoparticles. This can be demonstrated by SEM image of $\mathrm{Zn}-\mathrm{Ag}_{5}$.

Figure 2(a) shows the image of $\mathrm{ZnO}$ nanorods calcined at $450^{\circ} \mathrm{C}$, a large number of open-ended $\mathrm{ZnO}-\mathrm{NRs}$ with uniform diameters around $300 \mathrm{~nm}$ and lengths about several micrometer can be clearly seen in the picture. No obvious damage is found and the materials remain in good shape. Figure 2(b) shows that Ag-sensitized $\mathrm{ZnO}$ prepared by photoreduction does not cause any change in the morphology compared with pure $\mathrm{ZnO}$. But some newly formed small Ag particles with diameter a few nanometer on the surface of $\mathrm{ZnO}$ can be observed clearly.

The EDS spectrum (Figure 3) showed that the $\mathrm{Zn}-\mathrm{Ag}_{5}$ material include elements such as $\mathrm{Zn}, \mathrm{O}$ and $\mathrm{Ag}$. The quality of $\mathrm{Ag}$ content deduced from the EDS spectrum about 3\% indicate that $\mathrm{Ag}$ particles are losed in the reaction process.

The room temperature UV-Vis spectrum of undoped $\mathrm{ZnO}$ and $\mathrm{Zn}-\mathrm{Ag}_{5}$ are presented in Figure 3. As can be seen from curve A in Figure 4, $\mathrm{ZnO}-\mathrm{NRs}$ have a broad intense absorption below wavelength $400 \mathrm{~nm}$. It is the characteristic absorption of $\mathrm{ZnO}$ corresponding to the charge transfer process from the valence band to conduction band in $\mathrm{ZnO}$. In the $\mathrm{UV}-\mathrm{V}$ is spectra of the $\mathrm{Zn}-\mathrm{Ag}_{5}$ (curve B), it can be seen that there is a absorption peak

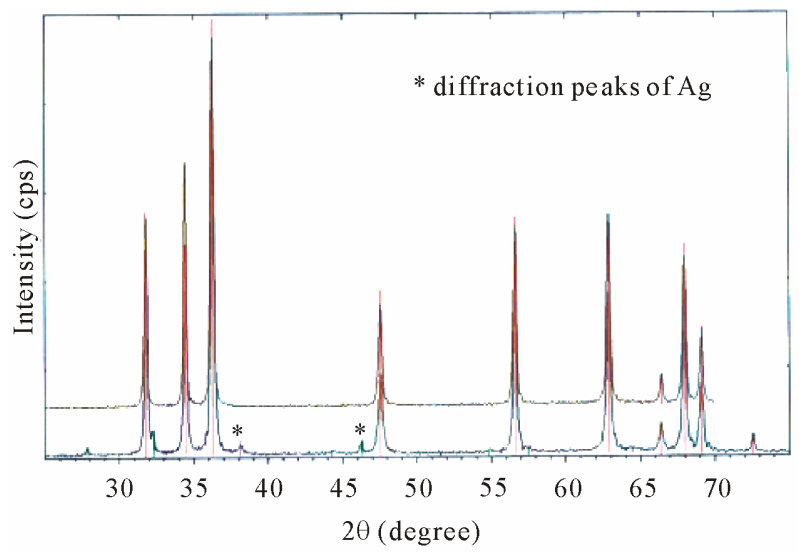

Figure 1. XRD patterns of $\mathrm{ZnO}$ and $\mathrm{Zn}-\mathrm{Ag}_{5}$.
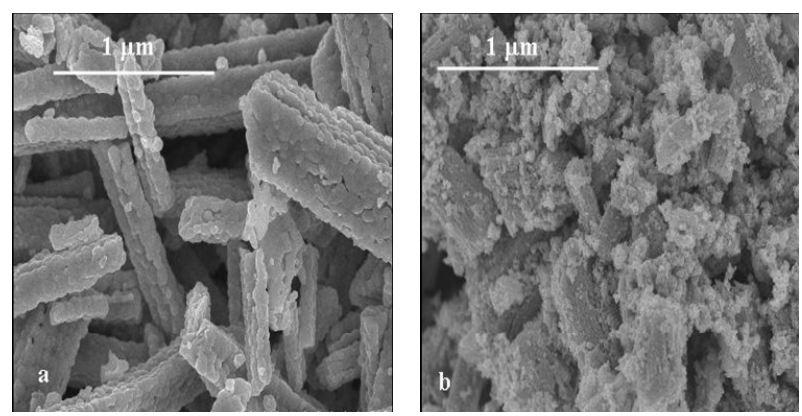

Figure 2. SEM images of $\mathrm{ZnO}$ nanorods (a) and $\mathrm{Zn}-\mathrm{Ag}_{5}$ (b). 


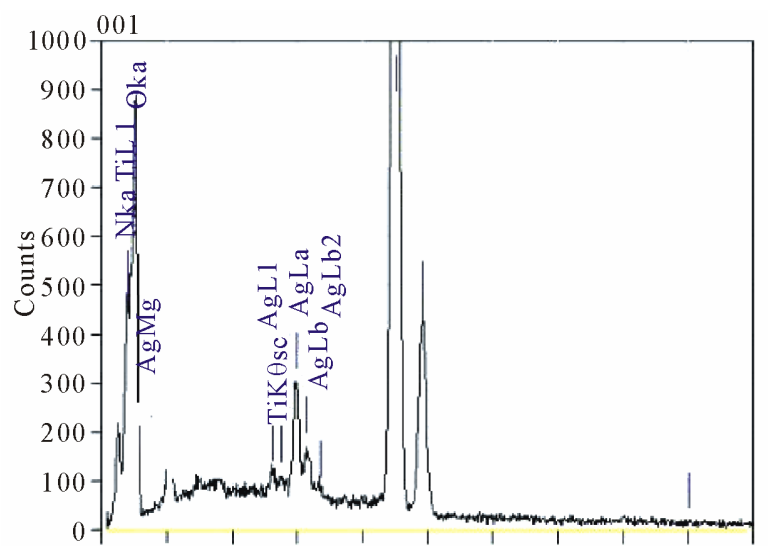

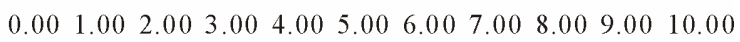
$\mathrm{keV}$

Figure 3. EDS spectrum of $\mathbf{Z n -} \mathbf{A g}_{5}$.

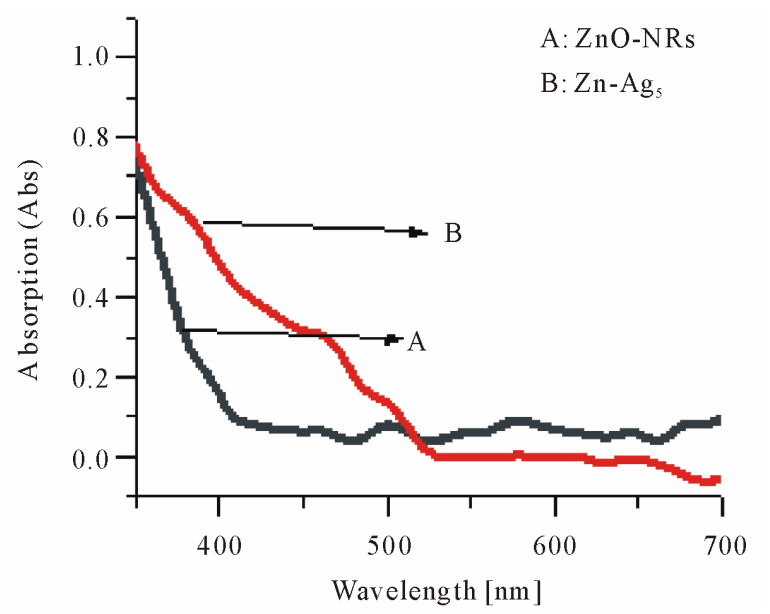

Figure 4. UV-Vis spectrums of $\mathrm{ZnO}$ and $\mathrm{Zn}-\mathrm{Ag}_{5}$.

around $460 \mathrm{~nm}$ in the visible range. This is the characteristic of surface plasmon absorption corresponding to $\mathrm{Ag}^{0}$ particles [9]. So the modification decrease in bandgap energy of $\mathrm{ZnO}$.

Figure 5 presents the room temperature Raman spectrum of $\mathrm{ZnO}-\mathrm{NRs}$ and $\mathrm{Zn}-\mathrm{Ag}_{5}$. It can be seen that the spectrum of $\mathrm{ZnO}$ (curve A) consists of five peaks located at about 100, 140, 380, 437, and $580 \mathrm{~cm}^{-1}$ which correspond to the fundamental phonon modes of hexagonal $\mathrm{ZnO}$, respectively. The curve $\mathrm{B}$ which is the spectrum of $\mathrm{Zn}-\mathrm{Ag}_{5}$ has four peaks located at about 100, 140, 241, and $580 \mathrm{~cm}^{-1}$. There appeared a broad Raman peak at about $241 \mathrm{~cm}^{-1}$ exclusively for the Ag-doped samples. The intensity of this peak decreased drastically on doping the silver in the samples. We can recall that the incorporation of $\mathrm{Ag}$ in our $\mathrm{ZnO}$ nanoparticles reduces their crystallinity [8].

Figure 6 shows room-temperature PL spectrums of $\mathrm{ZnO}$ and $\mathrm{Zn}-\mathrm{Ag}_{5}$ at the excitation wavelength of $325 \mathrm{~nm}$. We can see that a blue-green emissions at about $520 \mathrm{~nm}$

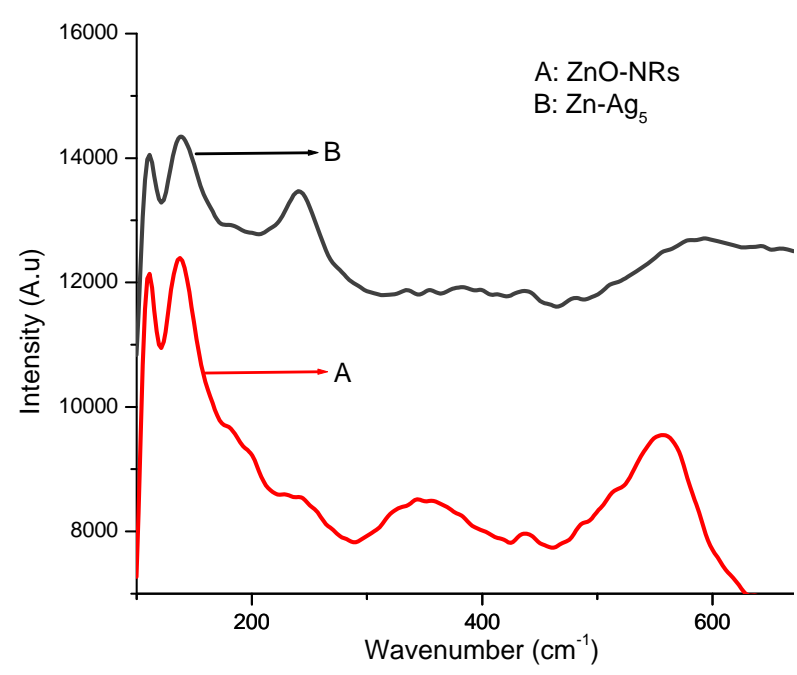

Figure 5. Raman spectrums of $\mathrm{ZnO}$ and $\mathrm{Zn}-\mathrm{Ag}_{5}$.

were observed in them. The emission centered at $520 \mathrm{~nm}$ of $\mathrm{Zn}-\mathrm{Ag}_{5}$, which can be fitted to two peaks centered at $516 \mathrm{~nm}$ and $630 \mathrm{~nm}$, respectively, as shown in the inset of Figure 5. The peak 516 might be attributed to the intrinsic defects $(\mathrm{O}$ and $\mathrm{Zn}$ vacancies or interstitials and their complexes) in $\mathrm{ZnO}$. The other hand, the Ag-doped $\mathrm{ZnO}$, which prepared, exhibits a new and unusual PL phenomenon at $615 \mathrm{~nm}$; there is the possibility of the formation of a new surface state. The dopant Ag has a great effect on the separation and recombination process of photo-induced charge carriers of $\mathrm{ZnO}$, which can further effect on PL performance. It indicates that the photoluminescence mechanism of $\mathrm{Ag} / \mathrm{ZnO}$ is very complex and further research is needed.

Figure 7 shows the effect of the Ag content on the photocatalytic activity of Ag-doped $\mathrm{ZnO}$. The photocatalytic degradation ratio of methyl blue (MB) increases rather rapidly initially with the increase of the Ag content and reaches a plateau at the $\mathrm{Ag}$ content of $4 \%$. It is worth that the amount of doped silver ion is very important to photoactivity. But an increase in dopant ion can make increasable rate recombination of electron-hole pairs, because silver ions play a central role of recombination, that can make decreasing the photocatalytic activity of material. The photocatalytic mechanism of $\mathrm{Ag} / \mathrm{ZnO}$ is also complex and it's studied deeply.

We considered the UV-photoreduction mechanisms from the view point of photolysis at $\mathrm{ZnO}$ catalyst. Oxidation and reduction occur at the same time in $\mathrm{Ag}$ ion photoreduction. In the reduction, the conduction band electrons generated in the $\mathrm{ZnO}\left(\mathrm{e}^{-}(\mathrm{CB})\right)$ by $\mathrm{UV}$ irradition can reduce adsorbed $\mathrm{Ag}^{+}$ions, giving rise to $\mathrm{Ag}$ atoms $\left(\mathrm{Ag}^{0}\right)$. The reduced $\mathrm{Ag}$ is deposited on the $\mathrm{ZnO}$ surface. Photochemical reactions induced by $\mathrm{ZnO}$-light are summarized as: 


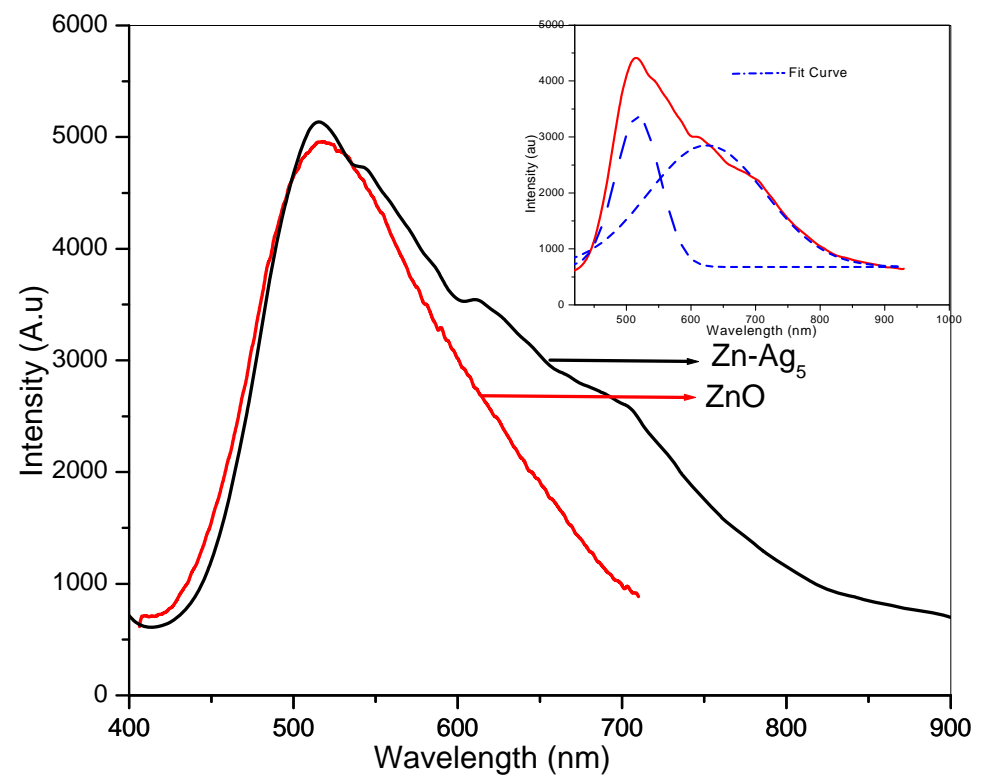

Figure 6. Room-temperature PL spectrum of $\mathrm{Zn}-\mathrm{Ag}_{5}$. In the inset, the curves “...”show Gaussian curve fitting.

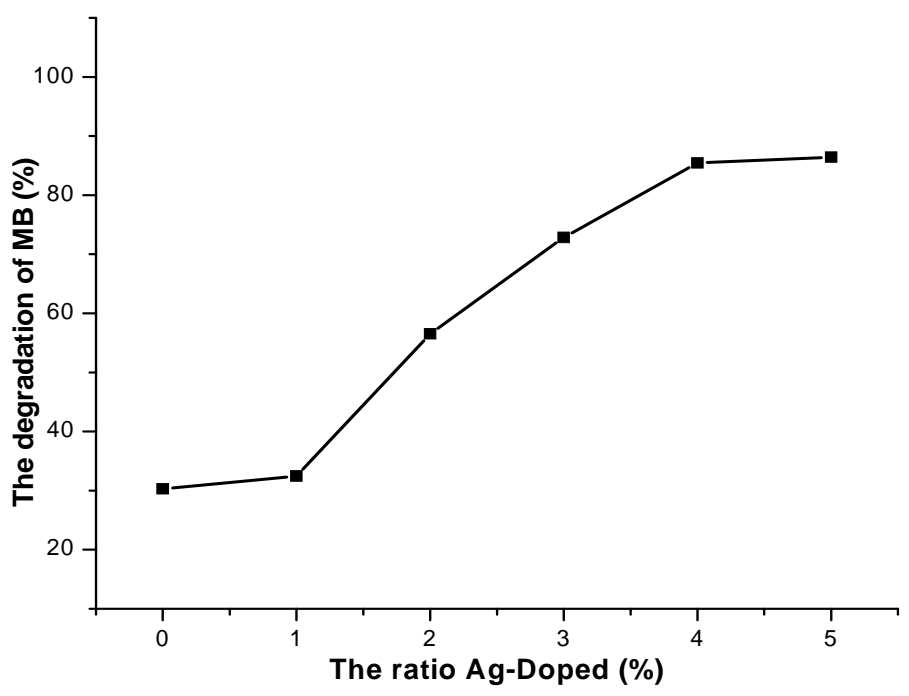

Figure 7. The degradation of MB on the Ag-doped ZnO.

$$
\begin{aligned}
& \mathrm{ZnO}+\mathrm{h} v \rightarrow \mathrm{e}^{-}(\mathrm{CB})+\mathrm{h}^{+}(\mathrm{VB}) \\
& \mathrm{Ag}^{+}+\mathrm{e}^{-}(\mathrm{CB}) \rightarrow \mathrm{Ag}^{0} \\
& 2 \mathrm{H}_{2} \mathrm{O}+\mathrm{h}^{+}(\mathrm{VB}) \rightarrow \mathrm{O}_{2}+4 \mathrm{H}^{+}
\end{aligned}
$$

\section{Conclusion}

Nanorod $\mathrm{ZnO}$ was successfully synthesized by a simple hydrothermal method. Ag-doped $\mathrm{ZnO}$ composites were prepared by method of UV-photoreduction with as-synthesized $\mathrm{ZnO}-\mathrm{NRs}$. Photocatalytic reactions show that doping $\mathrm{Ag}$ into $\mathrm{ZnO}$ hole remarkably improves the photocatalytic activity of $\mathrm{ZnO}$ under simulated solar light. The PL spectrum result shows that Ag-sensitized $\mathrm{ZnO}$ composite not only has the emission intensities at about $318 \mathrm{~nm}$ and $520 \mathrm{~nm}$, but also exhibits a new and unusual PL phenomenon at $615 \mathrm{~nm}$, indicating that the dopant $\mathrm{Ag}$ has great effects on separation and recombination processes of photo-induced charge carriers of $\mathrm{ZnO}$.

\section{REFERENCES}

[1] N. W. Emanetoglu, C. Gorla, Y. Liu, S. Liang and Y. Lu, "Epitaxial ZnO Piezoelectric Thin Films for Saw Filters," Materials Science in Semiconductor Processing, Vol. 2, No. 3, 1999, pp. 247-252. doi:10.1016/S1369-8001(99)00022-0

[2] Y. Chen, D. Bagnall and T. Yao, " $\mathrm{ZnO}$ as a Novel 
Photonic Material for the UV Region," Materials Science and Engineering: B, Vol. 75, No. 2-3, 2000, pp. 190-198. doi:10.1016/S0921-5107(00)00372-X

[3] P. Mitra, A. P. Chatterjee and H. S. Maiti, "ZnO Thin Film Sensor," Materials Letters, Vol. 35, No. 1-2, 1998, pp. 33-38. doi:10.1016/S0167-577X(97)00215-2

[4] G. Skanadan, Y. J. Chen, N. Glumac and B. H. Kear, "Synthesis of Oxide Nanoparticles in Low Pressure Flames," Nanostructured Materials, Vol. 11, No. 2, 1999, pp. 149-153. doi:10.1016/S0965-9773(99)00028-8

[5] T. Shinagawa, M. Izaki, H. Inui, K. Murase and Y. Awakura, "Characterization of Transparent Ferromagnetic Fe: $\mathrm{ZnO}$ Semiconductor Films Chemically Prepared from Aqueous Solutions," Journal of The Electrochemical Society, Vol. 152, No. 10, 2005, pp. 736-741. doi:10.1149/1.2001247

[6] R. Chen, C. Zou, J. Bian, A. Sandhu and W. Gao, "Mi- crostructure and Optical Properties Ag-Doped ZnO Nanostructures Prepare by a Wet Oxidation Doping Process," Nanotechnology, Vol. 22, No. 10, 2011, p. 1

[7] S. H. Jeong, B. N. Park, S. B. Lee and J.-H. Boo, "Structural and Optical Properties of Silver-Doped Zinc Oxide Sputtered Films," Surface \& Coatings Technology, Vol. 193, No. 1-3, 2005, pp. 340-344. doi:10.1016/j.surfcoat.2004.08.112

[8] B. D. Ahn, H. S. Kang, J. H. Kim, G. H. Kim, H. W. Chang and S. Y. Lee, "Synthesis and Analysis of AgDoped ZnO," Journal of Applied Physics, Vol. 100, 2006, Article ID: 093701. doi:10.1063/1.2364041

[9] K. Patel, S. Kapoor, D. P. Dave and T. Mukherjee, "Synthesis of Nanosized Silver Colloids by Microwave Dielectric Heating," Journal of Chemical Sciences, Vol. 117, No. 1, 2005, pp. 53-60. 